\title{
Analysis of transient laminar mass transfer in a parallel-plate dialyser
}

\author{
Spas D Kolev ${ }^{1, *}$ and Willem E van der Linden \\ Laboratory for Chemical Analysis, Department of Chemucal Technology, University of Twente, PO Box 217, \\ NL-7500 AE Enschede (Netherlands)
}

(Received 22nd April 1991, revised manuscript recerved 15th October 1991)

\begin{abstract}
Mass transfer under lamınar flow conditions is discussed on the basis of the Navier-Stokes equations and the axially dispersed plug-flow model By fitting the results obtained with these two approaches, relationshyps can be derived for predictıng the mass-transfer coefficient and the Peclet number in a parallel-plate laminar flow system with one impermeable wall and an opposite wall at which the concentration is uniform This single-stream relationship can be utilized for calculating the mass-transfer coefficients in both channels of a co-current parallel-plate dialyser It is shown that the Peclet number in the axially dispersed plug-flow model derived for a parallel-plate flow where both sides are impermeable gives better results when applied to the co-current dialyser than the single-stream relationship The results obtained allow the mathematical modelling of process and analytical flow-through manifolds incorporating a dialysis module
\end{abstract}

Keywords Flow system, Dialysis, Mass transfer, Mathematical modellıng

Flow-mjection manifolds incorporating modules for dialysis separation have been successfully introduced in analytical practice Among the various types of dialysers used in industry and in medical practice ( 1 e, parallel-plate, tubular and spiral-plate dialysers), the co-current parallelplate dialysers have been used most frequently in flow-injection manifolds so far For this reason this type of dialysis module will be considered here The Reynolds number in them is well below the limit of turbulence so that laminar flow can be expected to prevall

Even the simplest manifolds for flow-injection analysis consist of different flow-through elements (e g, straight, colled, knitted or packed-bed tubes, injection valves, pumps and flow-through

I Permanent address Faculty of Chemistry, Unversity of Sofia, Anton Ivanov Ave 1, BG-1126 Sofia, Bulgaria measurement cells with vanious geometries), which makes their mathematical description based on first principles (e g, Navier-Stokes equations) exceedingly difficult and in most instances virtually impossible For this reason their mathemat1cal modelling has usually been based on different hydraulic models (e $\mathrm{g}$, the tanks-ın-series model and the axially dispersed plug-flow mode) extensively used in chemical engineering [1,2] Among them the use of the axially dispersed plug-flow model proved to be very successful [3-7] Crucial parameters of this model when applied to a parallel-plate dialyser are the Peclet numbers and the mass-transfer coefficients in both channels of the dialyser

Instead of the heat- or mass-transfer coefficients, usually the corresponding Nusselt or Sherwood numbers are used. They represent the d1mensionless temperature or concentration gradients averaged over the heat- or mass-transfer 
surface (e g, membrane/flow interface) It should be taken into account that owing to the similarity of heat and mass transfer it is always possible to write a mass-transfer relationship if the corresponding heat-transfer relationship is available, or vice versa, provided that mass transfer takes place at low concentrations so that Fick's law holds

Attempts to calculate the global Nusselt or Sherwood number by the equation derived for heat conduction through composite walls [8] have been made $[9,10]$ King [11,12] showed that the applicability of this equation in multi-stream problems is limited by several strungent conditions which are rarely met in common mass-transfer geometries Szekely [13] showed that even in simple multi-phase transient mass-transfer problems such as transfer across the interface of two independently stirred liquids, non-additivity of resistances can be observed This shows that despite the obvious similarity between the well studied single-stream heat or mass transfer and the multi-stream problem, direct application of quantitative relationships derived for the former instance to the latter may lead to erroneous results and should only be used with great care

However, Nunge et al [14] showed, by comparing the steady-state values of the Nusselt numbers of the mdividual streams in a co-current heat exchanger with those for parallel-plate laminar flow with one side insulated and a uniform wall temperature boundary condition on the other side, that single-stream results can be used to predict co-current flow behaviour only when the two streams are very similar with respect to their characteristics ( $1 \mathrm{e}$, almost equal heights of the channels and very close values of the thermal conductivities and capacities of both streams)

Unlike dialysers used in industry and for medical purposes, on-line analytical dialysers recently incorporated in flow-injection systems operate under dynamic conditions This fact complicates drastically the problem of finding a theoretical relationship for calculating the mass-transfer coefficients (Sherwood numbers) in parallel-plate dialysers with lamınar flow in both channels No solutions to this problem have been found in the literature
The problem of calculating the other two empirical parameters, $1 \mathrm{e}$, the Peclet numbers of the donor and the acceptor streams, also has not yet been solved Only in the simpler case of a parallel-plate laminar flow with two impermeable sides has a theoretical relationship for calculating the relevant Peclet number been derived [15]

The objective of this investigation was to derive relationships for calculating the mass-transfer coefficients (Sherwood numbers) and the Peclet numbers in both channels of a parallelplate dialyser under laminar flow conditions

POSSIBLE APPROACHES FOR THE SOLUTION OF THE PROBLEM

Because of the complexity of the mathematical description of the transient behaviour of a parallel-plate dialyser based on first principles, no general analytical solutions for calculating the concentrations in the two channels are avallable that could be used further for deriving the relationships for determining the corresponding transfer coefficients One of the possible approaches for overcoming such difficulties, which is often utilized in chemical engineering, is to find a regression type of relationship between the quantities of interest and the dimensionless groups in the differential equations describing the dialyser For this purpose the equations mentioned above must be solved numerically for different values of their dimensionless groups and the results obtained should be processed for determining the corresponding mass-transfer coefficients (Sherwood numbers) in the framework of the model based on the axually dispersed plug-flow model However, in complicated systems such as that discussed here, the practical realization of this general approach will be accompanied by severe difficulties originating mainly from the large number of dimensionless groups governing the performance of the system An alternative approach which bypasses most of these difficulties is necessary It could be based on the utilization of single-stream results in analogy with the 
case of steady-state heat exchange [14] discussed above

SINGLE-STREAM PARALLEL-PLATE MASS TRANSFER

The transient mass-transfer process in a parallel-plate laminar flow with one impermeable wall and constant concentration at the opposite wall can be described using both the convective-diffusion equation (Eqn 1) or the axially dispersed plug-flow model (Eqn 2) (the symbols and their definitions are given in Table 1)

$$
\frac{\partial C}{\partial \theta}=\tau\left(\beta \frac{\partial^{2} C}{\partial X^{2}}+\frac{\partial^{2} C}{\partial Y^{2}}\right)-\frac{3}{2} Y(2-Y) \frac{\partial C}{\partial X}
$$

where

$C(\theta, X, Y)=\left[c(\theta, X, Y)-c_{\mathrm{w}}\right] /\left[c_{\mathrm{o}}-c_{\mathrm{w}}\right]$

The initial and boundary conditions of Eqn 1 are $C(0, X, Y)=0$ for $X>0$

$C(\theta, 0, Y)= \begin{cases}10 & \text { for } \theta \geqslant 0 \\ 00 & \text { for } \theta<0\end{cases}$

$C(\theta, \infty, Y)=0$

$\frac{\partial C(\theta, X, 0)}{\partial Y}=0$

$C(\theta, X, 2)=0$

$\frac{\partial \hat{C}}{\partial \theta}=\frac{1}{P} \frac{\partial^{2} \hat{C}}{\partial X^{2}}-\frac{\partial \hat{C}}{\partial X}-K \hat{C}$

where

$\hat{C}=\left(\hat{c}-c_{\mathrm{w}}\right) /\left(\hat{c}_{\mathrm{o}}-c_{\mathrm{w}}\right)$

The initial and boundary conditions of Eqn 2 are

$\hat{C}(0, X)=0$ for $X>0$

$\hat{C}(\theta, 0)=\left\{\begin{array}{lll}10 & \text { for } \theta \geqslant 0 \\ 00 & \text { for } \theta<0\end{array}\right.$

$\hat{C}(\theta, \infty)=0$

The average overall logarithmic steady-state Sherwood number used in the literature for characterizing steady-state mass transfer is usually related to the outlet mean concentration in the cross-section of the flow $(X=1)$ by the following equation [16]

$S h=\frac{-\ln \hat{C}}{(\tau / 4)}$

Takıng into consideration that by definition $S h=4 K / \tau$, one can write

$K=-\ln \hat{C}$

Equation 4 is in fact the steady-state solution of Eqn 2 at infinitely high Peclet number, 1 e, plug flow without axial dispersion

However, in practical flow systems the Peclet number has a finite value and the outlet concentration will depend not only on the steady-state mass-transfer coefficient $(K)$, or the corresponding Sherwood number, but also on the Peclet number as can be seen from the steady-state solution of Eqn 2

$\ln \hat{C}=P\left[05-(025+K / P)^{1 / 2}\right]$

For checking the possibility of using the steady-state value of $K$ (Eqn 5) for describing also the transient part of the concentration-time curve $\hat{C}(\theta, 1)$, Eqn 2 must be solved By Laplace transforms this linear partial differential equation can be reduced to a homogeneous ordinary differential equation of the second order having the following analytical solution in the Laplace domain

$\bar{C}=\frac{1}{p} \exp \left(P\left\{05-[025+(p+K) / P]^{1 / 2}\right\} X\right)$

The inverse Laplace transformation [17] of Eqn 6 gives the final tıme domain solution, which for $X=1$ has the form

$$
\begin{aligned}
\hat{C}= & \frac{1}{2} \exp (P / 2)\left\{\exp \left[-(\omega P)^{1 / 2}\right]\right. \\
& \times \operatorname{erfc}\left[\frac{1}{2}(P / \theta)^{1 / 2}-(\omega \theta)^{1 / 2}\right] \\
& +\exp \left[(\omega P)^{1 / 2}\right] \\
& \left.\times \operatorname{erfc}\left[\frac{1}{2}(P / \theta)^{1 / 2}+(\omega \theta)^{1 / 2}\right]\right\}
\end{aligned}
$$

where $\omega=P / 4+K$ 


\section{TABLE 1}

Symbols and definitions a

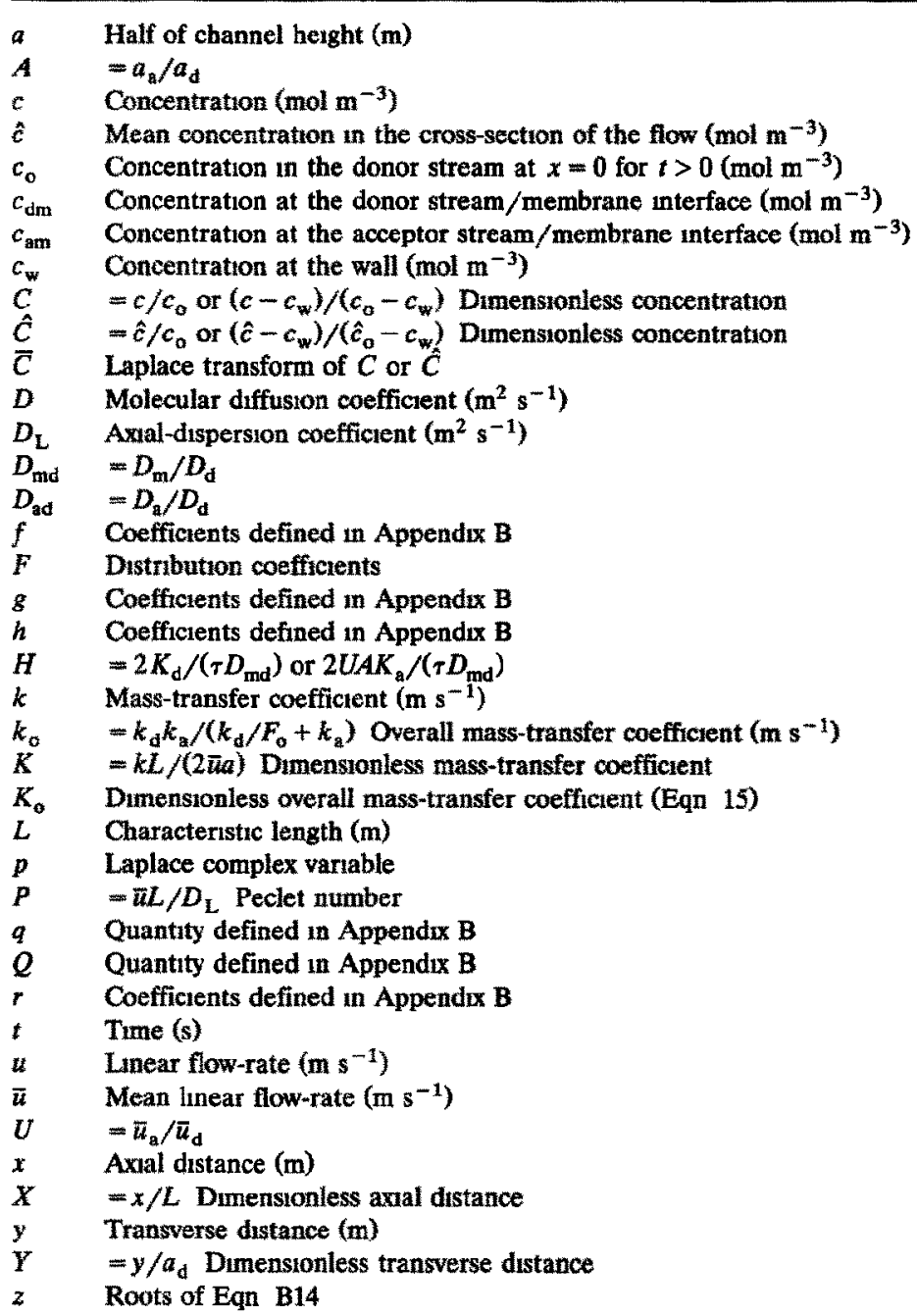

\section{Greek letters}

$\begin{array}{ll}\alpha & \text { Coefficients defined in Appendix B } \\ \beta & =\left(a_{\mathrm{d}} / I\right)^{2} \\ \delta & \text { Half-width of the membrane }(\mathrm{m}) \\ \Delta & =\delta / a_{\mathrm{d}} \text {. Dimenstonless half-with of the membrane } \\ \theta & =t \bar{u}_{\mathrm{d}} / L \text { Dimensionless time } \\ \tau & =D_{\mathrm{d}} L /\left(\tilde{u}_{\mathrm{d}} a_{\mathrm{d}}^{2}\right) \text { Founer number of the donor stream } \\ \omega & \text { Coefficient defined in Eqn } 7\end{array}$

a Subscripts $\mathrm{d}, \mathrm{a}$ and $\mathrm{m}$ refer to the donor stream, the acceptor stream and the membrane, respectively, $o$ and loc refer to overall and local, respectrvely 
The actual $\hat{C}(\theta, 1)$ curve was determined by solving Eqn 1 for Fourier numbers $(\tau)$ varying in the range 10-40 The solution of Eqn 1 was obtained by the numerical technique outlined in [15] The steady-state concentrations at $X=10$ were used for calculating the mass-transfer coefficients according to plug-flow (Eqn 4) and axlally dispersed plug-flow (Eqn 5) assumptions In the latter instance it is obvious that there is an infinite number of pairs of $P$ and $K$ values which will satisfy a given steady-state concentration (Eqn 5) For deciding which one of them should be preferred for a given value of the Fourier number, a parameter identification procedure for determining the values of $P$ and $K$ corresponding to the best fit between the concentration-time curves calculated by Eqns 1 and 2 should be performed A simplex optımization method based on the algorthm of Nelder and Mead was uttlized [18] The function minimized by this method was the square root of the mean squared error between the two concentration-time curves mentioned above The results obtained showed that both $P$ and $K$ are linearly dependent on the Fourier number and can be calculated very precisely by Eqns 8 and 9, respectively

$P=4015 \tau$

$K=0175+0614 \tau$
The agreement between the transient parts of the $\hat{C}(\theta, 1)$ curves calculated by the convectivediffusion equation (Eqn 1) and the axially dispersed plug-flow model (Eqn 2), if Eqns 8 and 9 are used, is very good and for $\tau>15$ both curves are virtually indistınguishable For lower $\tau$ values there is a slight deviation in the lower concentration part of the curves (Fig 1a)

The relationshup between the Peclet and the Fourier numbers is of the same type as that derived for the case of two impermeable plates [15]

$P=525 \tau$

However, the coefficient in Eqn 8 is lower than that in Eqn 10, showing more pronounced dispersion This effect is the result of the zero concentration boundary condition at one of the plates which enhances the transverse concentration inhomogeneity in the flow Sumulations of Eqn 2 in which $K$ and $P$ were varied showed that the steady-state concentration is determined mainly by the mass-transfer coefficient This conclusion is supported also by the simulations of Bernhardsson et al [19], who showed that under steady-state conditions the substitution of the parabolic velocity profile in the convective-diffusion equation with a flat profile will only slightly affect the overall mass-transfer process in a co-

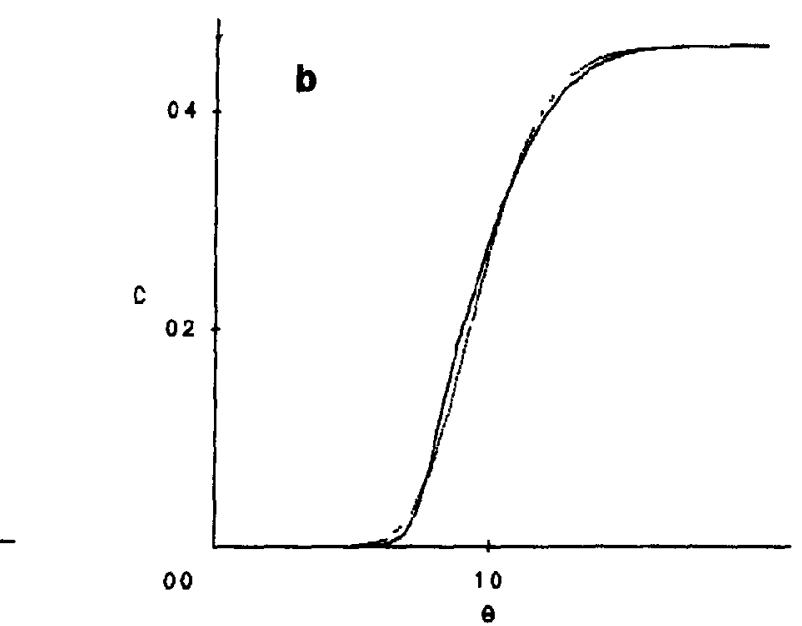

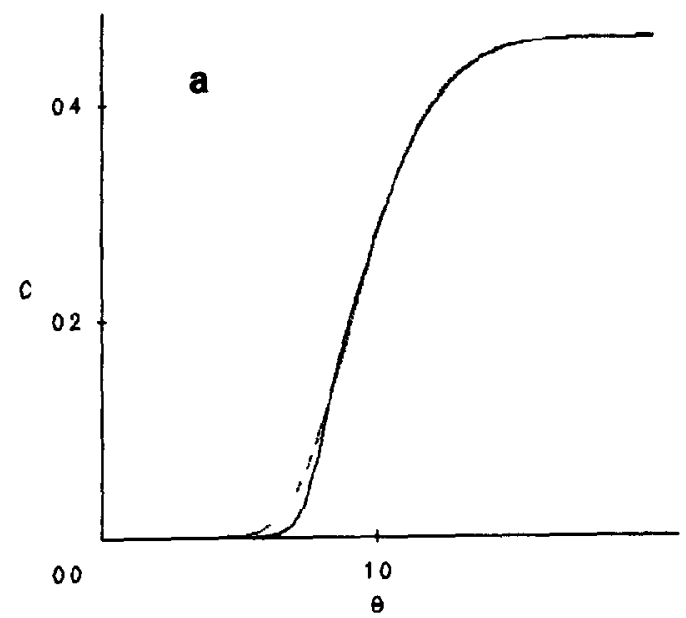

Fig 1 Concentration profiles calculated by the convective-diffusion equation (solid line) and the axially dispersed plug-flow model (dotted line) on the basis of (a) Eqn (8) and (b) Eqn (10) 
TABLE 2

Values of the Sherwood number calculated for different values of the Fourner number $(\tau)$

\begin{tabular}{lllll}
\hline$S h$ & $\tau$ & & & \\
\cline { 2 - 5 } & 10 & 20 & 30 & 40 \\
\hline$S h(P=525 \tau)$ & 3148 & 2810 & 2696 & 2630 \\
$S h(P \rightarrow \infty)$ & 3094 & 2762 & 2652 & 2596 \\
$S h[20]$ & 2850 & 2642 & 2573 & 2539 \\
\hline
\end{tabular}

current parallel-plate dialyser The influence of the Peclet number can be observed in the transient part of $\hat{C}(\theta, 1)$ For this reason, if it is assumed that the value of the Peclet number does not change because of the mass transfer (Eqn $10)$, the error in calculating the steady-state concentration will be negligible while at the same tume for lower $\tau$ values more pronounced deviations in the transient parts of the curves will be observed (Fig 1b) However, for high $\tau$ values (e $g, \tau>3$ ) even the transient concentration can be predicted successfully under this assumption

The values of the logarithmic average Sherwood number for the parallel-plate system considered above, determined in the case of plug flow $(P \rightarrow \infty)$ and axially dispersed plug flow were compared with the solution of Hatton and Quarmby [20] for the local Nusselt number in a parallel-plate flow with one side insulated and constant temperature on the other The calculation of the local Nusselt number was based on the first ten eigenvalues presented in their paper Because of the complete simılarity between heat and mass transfer, instead of the Nusselt number, the Sherwood number will be used to avold confusion when the results in [20] are referred to The logarithmic average Sherwood number $(S h)$ for a given Fourier number $(\tau)$ was calculated on the basis of the local Sherwood number $\left(S h_{10 c}\right)$ [20] by

$S h=\frac{1}{\tau} \int_{0}^{\tau} S h_{10 c} \mathrm{~d} \tau$

The results presented in Table 2 show that if the axial dispersion is taken into consideration the values of the Sherwood numbers are slightly higher in comparison with those for $P \rightarrow \infty$ The Sh values according to Hatton and Quarmby [20] corresponding to the assumption $P \rightarrow \infty$ are within 2-8\% lower than the values calculated in this study for the same $\tau$ values For higher $\tau$ values the agreement improves considerably (e $g$, for $\tau=4$ the error is $-220 \%$ ), which suggests that for lower $\tau$ values more than ten eigenvalues are necessary for calculatıng the corresponding Sherwood (Nusselt) number if better precision is required

\section{CO-CURRENT DOUBLE-STREAM PARALLEL-PLATE MASS TRANSFER}

Such a system can be assumed to consist of three parallel plates The outer two are totally impermeable whereas the middle one separating the donor and the acceptor streams is permeable to the solute A step-function input at $x=0$ (Fig 2) is assumed to take place in the donor stream The other assumptions on which the model is based are outlined elsewhere [21]

As in the single-stream case this system can be described both by using the Navier-Stokes equations or the axially dispersed plug-flow model A model based on first principles by utilizing the former approach together with its solution is described in detall in [21] The latter approach provides a much simpler description of a co-current laminar flow dialyser The corresponding

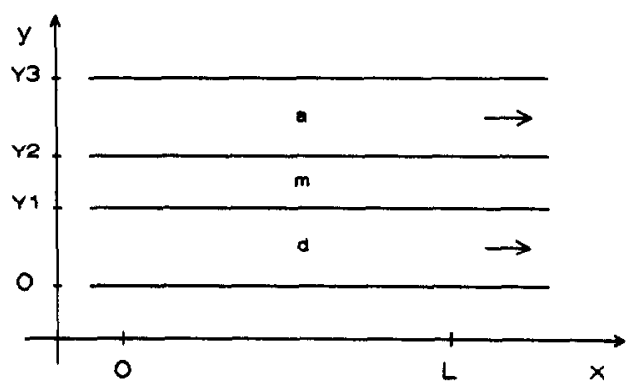

Fig 2 Scheme of the co-current flow system $Y 1=2 a_{\mathrm{d}}$, $Y 2=2\left(a_{\mathrm{d}}+\delta\right), Y 3=2\left(a_{\mathrm{d}}+\delta+a_{\mathrm{a}}\right), \mathrm{d}, \mathrm{m}$ and a refer to the donor stream, the membrane and the acceptor stream, respectively 
model in dimensionless quantities and variables with the appropriate initial and boundary cond1tions will take the following form

$$
\begin{aligned}
& \frac{\partial \hat{C}_{\mathrm{d}}}{\partial \theta}=\frac{1}{P_{\mathrm{d}}} \frac{\partial^{2} \hat{C}_{\mathrm{d}}}{\partial X^{2}}-\frac{\partial \hat{C}_{\mathrm{d}}}{\partial X}-K_{\mathrm{d}}\left(\hat{C}_{\mathrm{d}}-C_{\mathrm{dm}}\right) \\
& \frac{\partial C_{\mathrm{m}}}{\partial \theta}=D_{\mathrm{md}} \tau \frac{\partial^{2} C_{\mathrm{m}}}{\partial Y^{2}} \\
& \frac{1}{U} \frac{\partial \hat{C}_{\mathrm{a}}}{\partial \theta}=\frac{1}{P_{\mathrm{a}}} \frac{\partial^{2} \hat{C}_{\mathrm{a}}}{\partial X^{2}}-\frac{\partial \hat{C}_{\mathrm{a}}}{\partial X}+K_{\mathrm{a}}\left(C_{\mathrm{am}}-\hat{C}_{\mathrm{a}}\right) \\
& \hat{C}_{\mathrm{d}}(0, X)=C_{\mathrm{m}}(0, Y)=\hat{C}_{\mathrm{a}}(0, X)=0 \\
& \quad \text { for } X>0 \\
& \hat{C}_{\mathrm{d}}(\theta, 0)=\left\{\begin{array}{l}
10 \quad \text { for } \theta \geqslant 0 \\
00
\end{array} \quad \text { for } \theta<0, \quad \hat{\mathrm{a}}(\theta, 0)=0\right. \\
& \hat{C}_{\mathrm{d}}(\theta, \infty)=\hat{C}_{\mathrm{a}}(\theta, \infty)=0 \\
& F_{\mathrm{d}} C_{\mathrm{dm}}(\theta, X)=C_{\mathrm{m}}(\theta, 2) \\
& H_{\mathrm{d}}\left(\hat{C}_{\mathrm{d}}-C_{\mathrm{dm}}\right)=-\partial C_{\mathrm{m}}(\theta, 2) / \partial Y \\
& F_{\mathrm{a}} C_{\mathrm{am}}(\theta, X)=C_{\mathrm{m}}(\theta, 2+2 \Delta) \\
& H_{\mathrm{a}}\left(C_{\mathrm{am}}-\hat{C}_{\mathrm{a}}\right)=-\partial C_{\mathrm{m}}(\theta, 2+2 \Delta) / \partial Y
\end{aligned}
$$

The original equations are given in Appendix $\mathbf{A}$ and the solution of Eqns $12-14$ is presented in Appendix B

For checking the hypothesis of the applicability of single-stream results for the double-stream case, it seemed expedient to introduce some additional simplifications in order to reduce the number of unknown parameters in the model

A special case of the flow system discussed above which is of substantial practical signifcance occurs when the resistance of the membrane to the mass-transfer process is negligible Such a situation can be encountered when the membrane is much thinner than the height of the channels ( $\mathrm{e}, \Delta \rightarrow 0$ ) and the diffusion coefficient of the solute in it is not much lower than the corresponding value in the fluids on both sides Obviously these conditions hold farly often in dialysers used in practice The mathematical description of such a dialyser no longer requires the diffusion equation (Eqn 13) for the mem- brane The compatibility boundary conditions of Eqns 12 and 14 in such a case are

$$
\begin{aligned}
& F_{\mathrm{o}} C_{\mathrm{d}}(\theta, X, 2)=C_{\mathrm{a}}(\theta, X, 2) \\
& \frac{\partial C_{\mathrm{d}}(\theta, X, 2)}{\partial Y}=D_{\mathrm{ad}} \frac{\partial C_{\mathrm{a}}(\theta, X, 2)}{\partial Y}
\end{aligned}
$$

where $F_{\mathrm{o}}=F_{\mathrm{d}} / F_{\mathrm{a}}$ is the overall distribution coefficient of a given solute between the two liquid phases on both sides of the membrane

The number of parameters in the model in such a case can be reduced by one by introducing an overall mass-transfer coefficient, $K_{0}$ (Eqn 15), defined with respect to the parameters of the donor stream, instead of the individual coefficients for both streams ( $\mathrm{e}, K_{\mathrm{d}}$ and $K_{\mathrm{a}}$ )

$K_{\mathrm{o}}=\frac{K_{\mathrm{d}} K_{\mathrm{a}}}{K_{\mathrm{a}}+F_{\mathrm{o}} K_{\mathrm{d}} /(U A)}$

In dimensionless quantities and variables the mass-transfer equations for the donor and the acceptor stream will be the following

$$
\begin{aligned}
& \frac{\partial \hat{C}_{\mathrm{d}}}{\partial \theta}=\frac{1}{P_{\mathrm{d}}} \frac{\partial^{2} \hat{C}_{\mathrm{d}}}{\partial X^{2}}-\frac{\partial \hat{C}_{\mathrm{d}}}{\partial X}-K_{\mathrm{o}}\left(\hat{C}_{\mathrm{d}}-\hat{C}_{\mathrm{a}} / F_{\mathrm{o}}\right) \\
& \frac{1}{U} \frac{\partial \hat{C}_{\mathrm{a}}}{\partial \theta}=\frac{1}{P_{\mathrm{a}}} \frac{\partial^{2} \hat{C}_{\mathrm{a}}}{\partial X^{2}}-\frac{\partial \hat{C}_{\mathrm{a}}}{\partial X}+\frac{K_{\mathrm{o}}}{U A}\left(\hat{C}_{\mathrm{d}}-\hat{C}_{\mathrm{a}} / F_{\mathrm{o}}\right)
\end{aligned}
$$

In connection with the evaluation of the Peclet numbers in both streams, it should be taken into consideration that the zero concentration and the zero flux (1 e , impermeable wall) boundary cond1tions in the single-stream flow, considered earlier, create two extreme flow patterns, corresponding to the maximum and munumum possible transverse non-uniformity of the concentration in a parallel-plate flow under laminar conditions The values of the Peclet numbers in the doublestream case should lie between the values corresponding to the extreme cases mentioned above, $1 \mathrm{e}, 4015 \tau-525 \tau$ A comparison between the transverse distribution of the concentration in the donor stream ( $\mathrm{Ftg} 3 \mathrm{a}$ ) with the single-stream distribution in the cases of one impermeable wall and constant concentration at the other ( $\mathrm{F} 1 \mathrm{~g} \mathrm{3b}$ ) and two impermeable walls ( $F_{1 g} 3 c$ ) shows that 
the double-stream mass transfer is much closer to the case of two impermeable walls than to the other extreme of constant concentration at one of the walls For this reason, an attempt was made to describe the parallel-plate dialyser with an infinitely thin membrane using the model based on the assumption of axially dispersed plug flow in which the Peclet numbers and $K_{0}$ were calcu- lated by Eqns. 10 and 15, respectively The model based on first principles [21] was solved for $A$, $D_{\text {ad }}, U$ and $F_{\text {ad }}$ in the range 02-5 while in each case $\tau_{\mathrm{d}}$ was varied from 10 to 30 It should be taken into account that $A, D_{\mathrm{ad}}$ and $U$ affect $K_{\mathrm{o}}$ not only directly, as can be seen from Eqn 15, but also indirectly through the Fourier number of the acceptor stream, $\tau_{\mathrm{a}}$, which differs from that of
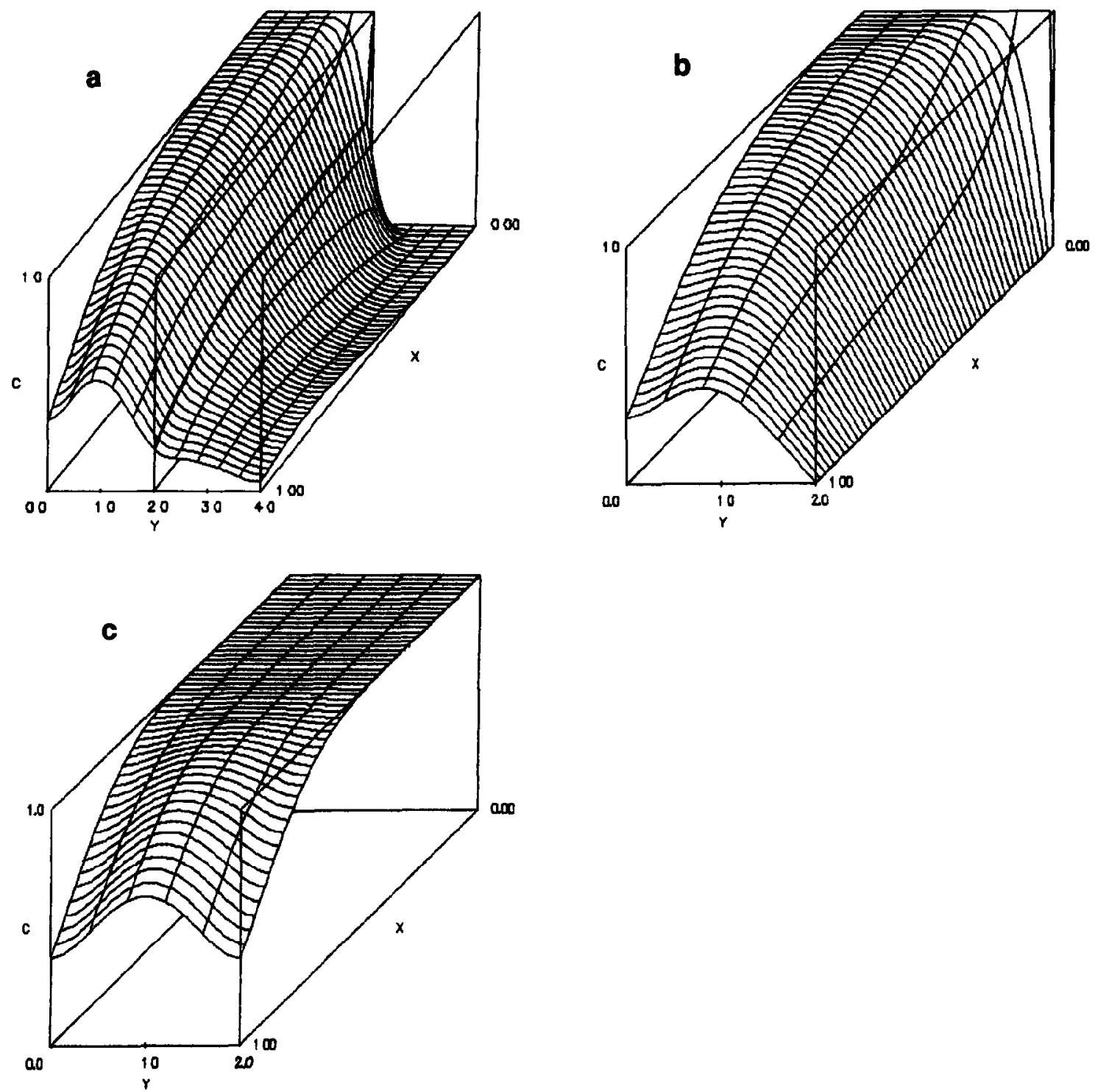

Fig 3 Spatial distribution of the solute in a parallel-plate dialyser in the case of $\Delta=0$ (a) and in a stngle-stream parallel-plate flow with one impermeable plate and constant concentration at the other (b) and with two impermeable plates $(c)$ at $\tau=10$ and $\theta=10$ 
the donor stream if one of the parameters devlates from unity. The agreement between the two models concerning the steady-state concentrations in both channels of the dialyser was good for all sets of parameter values even in the cases when $\tau_{a}$ was less than 10 This agreement is illustrated in Table 3 for different $D_{\text {ad }}$ values This result differs from the conclusions drawn in [14], which state that a necessary condition for ut1lizing single-stream results for double-stream co-current flow under steady-state conditions is almost identical properties of the two streams

The agreement between the transient concentrations in both channels of the dialyser calculated by the models mentioned above for $\tau_{d}=\tau_{a}$ $=10$ is illustrated in Fig 4 This agreement improves with increasing values of the Fourier numbers of the streams and for $\tau_{\mathrm{d}}=\tau_{\mathrm{a}}=30$ the concentration-time curves predicted by the two models become virtually indistinguishable If the other parameters of the dialyser $\left(\mathrm{e} g, A, D_{\text {ad }}, F_{\text {ad }}\right.$ and $U)$ are varied, then the smaller their deviation from unity the better is the agreement between the transient concentrations

Having established that Eqns 9 and 10 can be used successfully for calculating the mass-transfer coefficients and Peclet numbers of the individual streams in a parallel-plate dialyser with an infinitely thin membrane, their applicability in the case of a membrane with finite thickness should be investigated For this reason, the concentra-

\section{TABLE 3}

Comparison between the steady-state concentrations in the donor and acceptor streams calculated for $\tau_{d}=20$ and different $D_{\text {ad }}$ values by the model based on first principles (model 1) and on the assumption for axially dispersed plug flow (model 2)

\begin{tabular}{lllllll}
\hline$D_{\text {ad }}$ & \multicolumn{2}{l}{ Model 1 } & & \multicolumn{2}{c}{ Model 2 } & $\tau_{\mathrm{a}}$ \\
\cline { 2 - 3 } \cline { 5 - 6 } & Donor & Acceptor & & Donor & Acceptor & \\
\hline 020 & 07672 & 02328 & & 07662 & 02338 & 040 \\
050 & 06858 & 03142 & & 06859 & 03141 & 100 \\
075 & 06489 & 03511 & & 06489 & 03511 & 150 \\
100 & 06251 & 03749 & & 06252 & 03748 & 200 \\
125 & 06094 & 03906 & & 06089 & 03911 & 250 \\
150 & 05985 & 04015 & 05975 & 04025 & 300 \\
200 & 05840 & 04160 & 05828 & 04172 & 400 \\
500 & 05583 & 04417 & 05570 & 04494 & 1000 \\
\hline
\end{tabular}

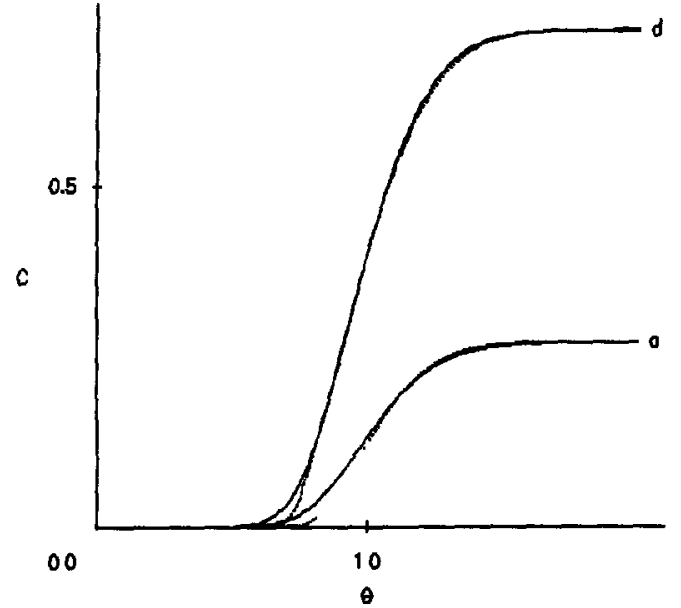

Fig 4 Concentration profiles calculated by the model assuming axially dispersed plug flow (solid hines) and the model based on first principles (dotted lines) for $\tau=10$ and $\Delta=0$ (d and $a$ refer to the donor and the acceptor streams, respectively)

tıon-tıme curves in both channels calculated by the model based on first principles [21] and the model utilizing the axially dispersed plug-flow model (Eqns 12-14) were compared In addition to the parameters varied in the case of $\Delta=0$, the two models mentioned above were compared also for different values of $D_{\mathrm{md}}, \Delta, F_{\mathrm{d}}$ and $F_{\mathrm{a}}$ The results obtained were similar to those for the case of $\Delta=0$, showing that Eqns 9 and 10 are also applicable in the general case In Fig 5 the satisfactory agreement between the two models for the extreme case of $\Delta=075$ is illustrated. For thinner membranes (1 e, lower $\Delta$ values), as can be expected, better agreement was observed The possibility of using single-stream results successfully for the description of the mass exchange in a parallel-plate dialyser shows the additivity of the mass transfer resistances of the individual phases This conclusion contradicts the results based on theoretical considerations of some very simplified multı-phase flow systems [11-13], according to which the additivity of resistances of the individual phases can be expected only if several stringent conditions hold Some of these conditions (e $\mathrm{g}$, the mass-transfer resistances must not interact [11]) are violated when the parallel-plate dialyser is considered Probably 


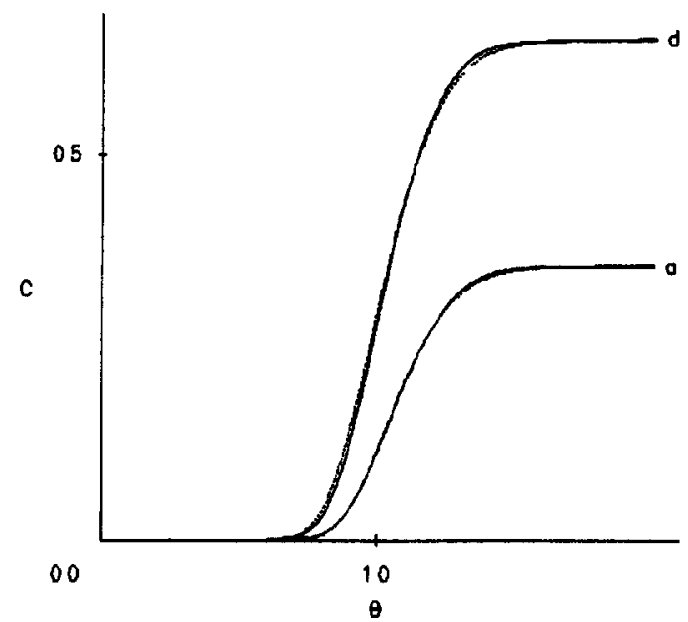

Fig 5 Concentration profiles calculated by the model assuming axially dispersed plug flow (solid lines) and the model based on first principles (dotted lines) for $\Delta=075$ and $\tau=20$ ( $d$ and a refer to the donor and the acceptor streams, respectively)

compensation of the errors caused by violation of these conditions takes place

\section{SOFTWARE}

The numerical procedures used were programmed in $\mathrm{C}$ and the corresponding programs were run on IBM/PC compatible and VAX computers

\section{Conclusions}

Relationships for calculatıng the mass-transfer coefficient and the Peclet number in a singlestream parallel-plate laminar flow with one impermeable side and uniform concentration on the other side were derived Considering the masstransfer process in a parallel-plate dialyser, it was found that the single-stream result mentioned above can also be used, thus showing the existence of additivity of the mass-transfer resistances of the individual phases of the dialyser It was found that the Peclet numbers in the dialyser can be successfully approximated by the theoret1cal equation obtained elsewhere for the case of two impermeable sides These results have shown that it is allowed to use the axially dispersed plug-flow assumption for the mathematical modelling of transient and steady-state mass-transfer processes in parallel-plate dialysers with fully developed laminar flow in both channels which find application in process and analytical flow-through systems In this way the serious calculation problems associated with the numerical solution of the convective-diffusion equation could be avoided The development of such a model for a flow-injection manifold with a parallel-plate dialysis module is in progress

\section{APPENDIX A}

Mathematical description of a co-current parallel-plate dialyser based on the axially dispersed plug-flow model

$$
\begin{aligned}
& \frac{\partial \hat{c}_{\mathrm{d}}}{\partial t}=D_{L_{\mathrm{d}}} \frac{\partial^{2} \hat{c}_{\mathrm{d}}}{\partial x^{2}}-\bar{u}_{\mathrm{d}} \frac{\partial \hat{c}_{\mathrm{d}}}{\partial x}-\frac{k_{\mathrm{d}}}{2 a_{\mathrm{d}}}\left(\hat{c}_{\mathrm{d}}-c_{\mathrm{dm}}\right) \\
& \frac{\partial c_{\mathrm{m}}}{\partial t}=D_{\mathrm{m}} \frac{\partial^{2} c_{\mathrm{m}}}{\partial y^{2}}
\end{aligned}
$$

$$
\frac{\partial \hat{c}_{\mathrm{a}}}{\partial t}=D_{L_{\mathrm{a}}} \frac{\partial^{2} \hat{c}_{\mathrm{a}}}{\partial x^{2}}-\bar{u}_{\mathrm{a}} \frac{\partial \hat{c}_{\mathrm{a}}}{\partial x}+\frac{k_{\mathrm{a}}}{2 a_{\mathrm{a}}}\left(c_{\mathrm{am}}-\hat{c}_{\mathrm{a}}\right)
$$

It should be taken into consideration that the concentration according to the axially dispersed plug-flow model corresponds to the mean concentration in the cross-section of the flow according to the diffusion-convection equation, $1 \mathrm{e}$

$$
\begin{aligned}
& \hat{c}_{\mathrm{d}}=\frac{1}{2 a_{\mathrm{d}}} \int_{0}^{2 a_{\mathrm{d}}} c_{\mathrm{d}}(t, x, y) \mathrm{d} y \\
& \hat{c}_{\mathrm{a}}=\frac{1}{2 a_{\mathrm{a}}} \int_{2\left(a_{\mathrm{d}}+\delta\right)}^{2\left(a_{\mathrm{d}}+\delta+a_{\mathrm{a}}\right)} c_{\mathrm{a}}(t, x, y) \mathrm{d} y
\end{aligned}
$$

The initial and boundary conditions are the following

$$
\begin{aligned}
& \hat{c}_{\mathrm{d}}(0, x)=c_{\mathrm{m}}(0, y)=\hat{c}_{\mathrm{a}}(0, x)=0 \text { for } x>0 \\
& \hat{c}_{\mathrm{d}}(t, 0)=\left\{\begin{array}{ll}
c_{\mathrm{o}} & \text { for } t \geqslant 0 \\
00 & \text { for } t<0
\end{array}, \hat{c}_{\mathrm{a}}(t, 0)=0\right. \\
& \hat{c}_{\mathrm{d}}(t, \infty)=\hat{c}_{\mathrm{a}}(t, \infty)=0 \\
& F_{\mathrm{d}} c_{\mathrm{dm}}(t, x)=c_{\mathrm{m}}\left(t, 2 a_{\mathrm{d}}\right)
\end{aligned}
$$


$k_{\mathrm{d}}\left(\hat{c}_{\mathrm{d}}-c_{\mathrm{dm}}\right)=-D_{\mathrm{m}} \frac{\partial c_{\mathrm{m}}\left(t, 2 a_{\mathrm{d}}\right)}{\partial y}$

$F_{\mathrm{a}} c_{\mathrm{am}}(t, x)=c_{\mathrm{m}}\left(t, 2 a_{\mathrm{d}}+2 \delta\right)$

$k_{\mathrm{a}}\left(c_{\mathrm{am}}-\hat{c}_{a}\right)=-D_{\mathrm{m}} \frac{\partial c_{\mathrm{m}}\left(t, 2 a_{\mathrm{d}}+2 \delta\right)}{\partial y}$

\section{APPENDIX B}

\section{Solution of Eqns 12-14}

These equations are linear partial differential equations of the second order with one temporal and one spatial variable In the Laplace domain they are transformed into the following ordinary differential equations

$$
\begin{aligned}
& \frac{\mathrm{d}^{2} \bar{C}_{\mathrm{d}}}{\mathrm{d} X^{2}}-P_{\mathrm{d}} \frac{\mathrm{d} \bar{C}_{\mathrm{d}}}{\mathrm{d} X}-P_{\mathrm{d}}\left(K_{\mathrm{d}}+p\right) \bar{C}_{\mathrm{d}}+P_{\mathrm{d}} K_{\mathrm{d}} \bar{C}_{\mathrm{dm}}=0 \\
& \frac{\mathrm{d}^{2} \bar{C}_{\mathrm{m}}}{\mathrm{d} Y^{2}}-\frac{p}{\tau D_{\mathrm{md}}} \bar{C}_{\mathrm{m}}=0 \\
& \frac{\mathrm{d}^{2} \bar{C}_{\mathrm{a}}}{\mathrm{d} X^{2}}-P_{\mathrm{a}} \frac{\mathrm{d} \bar{C}_{\mathrm{a}}}{\mathrm{d} X}-P_{\mathrm{a}}\left(K_{\mathrm{a}}+\frac{p}{U}\right) \bar{C}_{\mathrm{a}}+P_{\mathrm{a}} K_{\mathrm{a}} \bar{C}_{\mathrm{am}}=0
\end{aligned}
$$

The corresponding boundary conditions can be obtained from those of Eqns 12-14 by simply substituting the concentrations with their Laplace transforms, the only exception being

$\bar{C}_{\mathrm{d}}(0)=1 / p$

By solving Eqn B2 it is possible to express $\bar{C}_{\mathrm{dm}}$ and $\bar{C}_{\text {am }}$ in Eqns $B 1$ and $B 3$ as a function of $\bar{C}_{\mathrm{d}}$ and $\bar{C}_{\mathrm{a}}$

$\bar{C}_{\mathrm{dm}}=f_{\mathrm{d}} \overline{\mathrm{C}}_{\mathrm{d}}+f_{\mathrm{d}} \bar{C}_{\mathrm{a}}$

$\bar{C}_{\mathrm{am}}=g_{\mathrm{d}} \bar{C}_{\mathrm{d}}+g_{\mathrm{a}} \bar{C}_{\mathrm{a}}$

where

$$
\begin{aligned}
f_{\mathrm{d}}= & \frac{H_{\mathrm{d}}}{F_{\mathrm{d}} Q}\left[\left(q+H_{\mathrm{a}} / F_{\mathrm{a}}\right) \exp (2 q \Delta)+\left(q-H_{\mathrm{a}} / F_{\mathrm{a}}\right)\right. \\
& \times \exp (-2 q \Delta)] \\
f_{\mathrm{a}}= & \frac{H_{\mathrm{a}}}{F_{\mathrm{d}} Q} 2 q
\end{aligned}
$$

$$
\begin{aligned}
g_{\mathrm{d}}= & \frac{H_{\mathrm{d}}}{F_{\mathrm{a}} Q} 2 q \\
g_{\mathrm{a}}= & \frac{H_{\mathrm{a}}}{F_{\mathrm{a}} Q}\left[\left(q+H_{\mathrm{d}} / F_{\mathrm{d}}\right) \exp (2 q \Delta)+\left(q-H_{\mathrm{d}} / F_{\mathrm{d}}\right)\right. \\
& \times \exp (-2 q \Delta)] \\
Q= & \left(q+H_{\mathrm{d}} / F_{\mathrm{d}}\right)\left(q+H_{\mathrm{a}} / F_{\mathrm{a}}\right) \exp (2 q \Delta) \\
& -\left(q-H_{\mathrm{d}} / F_{\mathrm{d}}\right)\left(q-H_{\mathrm{a}} / F_{\mathrm{a}}\right) \exp (-2 q \Delta)
\end{aligned}
$$

$$
q=\left(p / \tau D_{\mathrm{md}}\right)^{1 / 2}
$$

After substituting $\bar{C}_{\text {md }}$ (Eqn B4) and $\bar{C}_{\text {ad }}$ (Eqn B5), the simultaneous differential Eqns B1 and B3 can be solved by an operational approach [22] The final solutions for $\bar{C}_{\mathrm{d}}$ and $\bar{C}_{\mathrm{a}}$ are

$\bar{C}_{\mathrm{d}}=\sum_{\imath=1}^{t=4} h_{\imath} \exp \left(z_{\imath}\right)$

$\bar{C}_{\mathrm{a}}=\sum_{i=1}^{t=4} r_{t} \exp \left(z_{i}\right)$

where $z_{a}$ are the roots of the following blquadratic equation

$$
\begin{aligned}
& z^{4}-\left(P_{\mathrm{d}}+P_{\mathrm{a}}\right) z^{3}+\left\{P_{\mathrm{d}} P_{\mathrm{a}}-\left[p+K_{\mathrm{d}}\left(1-f_{\mathrm{d}}\right)\right] P_{\mathrm{d}}\right. \\
& \left.\quad-\left[\frac{p}{U}+K_{\mathrm{a}}\left(1-g_{\mathrm{a}}\right)\right] P_{\mathrm{a}}\right\} z^{2} \\
& +P_{\mathrm{d}} P_{\mathrm{a}}\left[p\left(1+\frac{1}{U}\right)+K_{\mathrm{d}}\left(1-f_{\mathrm{d}}\right)+K_{\mathrm{a}}\left(1-g_{\mathrm{a}}\right)\right] z \\
& +P_{\mathrm{d}} P_{\mathrm{a}}\left\{\left[p+K_{\mathrm{d}}\left(1-f_{\mathrm{d}}\right)\right]\left[\frac{p}{U}+K_{\mathrm{a}}\left(1-g_{\mathrm{a}}\right)\right]\right. \\
& \left.\quad-K_{\mathrm{d}} K_{\mathrm{a}} f_{\mathrm{a}} g_{\mathrm{d}}\right\}=0
\end{aligned}
$$

which can be calculated analytically [23]

By substituting $\bar{C}_{\mathrm{d}}$ (Eqn B12) and $\bar{C}_{\mathrm{a}}$ (Eqn B13) in Eqns B1 and B3 and taking into consideration the linear independence of $\exp \left(z_{t}\right)$, it can easily be derived that

$r_{t}=\alpha_{\imath} h_{\imath}$

where

$\alpha_{i}=-\frac{z_{\imath}^{2}-P_{\mathrm{d}} z_{\mathrm{t}}-\left[p+K_{\mathrm{d}}\left(1-f_{\mathrm{d}}\right)\right] P_{\mathrm{d}}}{K_{\mathrm{d}} P_{\mathrm{d}} f_{\mathrm{a}}}$ 
The unknown integrational constants $h_{\imath}$ can be determined from the boundary conditions of Eqns B1 and B3

In the case of an infinitely thin membrane, the coefficients $f_{\mathrm{d}}, f_{\mathrm{a}}, g_{\mathrm{d}}$ and $g_{\mathrm{a}}$ can be determined by the following relationships

$f_{\mathrm{d}}=\frac{K_{\mathrm{d}}}{U A F_{\mathrm{o}} K_{\mathrm{a}}+K_{\mathrm{d}}}$

$f_{\mathrm{a}}=\frac{U A K_{\mathrm{a}}}{U A F_{\mathrm{o}} K_{\mathrm{a}}+K_{\mathrm{d}}}$

$g_{\mathrm{d}}=f_{\mathrm{d}} F_{\mathrm{o}}$

$g_{\mathrm{a}}=f_{\mathrm{a}} F_{\mathrm{o}}$

If instead of the individual mass-transfer coefficients the overall mass-transfer coefficient is used (1 e , Eqns 16 and 17), the final solution can be obtained in the same way as described above For this purpose the following substitutions in Eqn B14 must be performed

$K_{\mathrm{d}}=K_{\mathrm{o}}$

$K_{\mathrm{a}}=K_{\mathrm{o}} /(U A)$

$f_{\mathrm{d}}=0$

$f_{\mathrm{a}}=1 / F_{\mathrm{o}}$

$g_{\mathrm{d}}=1$

$g_{\mathrm{a}}=\mathbf{1}-\left(1 / F_{\mathrm{o}}\right)$

The numerical inverse Laplace transformation of $\bar{C}_{d}$ (Eqn B12) and $\bar{C}_{a}$ (Eqn B13) was performed by their expansion in Fourier sine series and subsequent analytical inverse transformation of these series $[24,25]$

\section{REFERENCES}

$1 \mathrm{~J}$ Ruzıcka and EH Hansen, Flow Injectıon Analysis, Wley, New York, 2nd edn , 1988
2 M Valcárcel and M D Luque de Castro, Flow-Injectıon Analysis Principles and Applications, Horwood, Chichester, 1987

3 J M Rein, WE van der Landen and H Poppe, Anal Chım Acta, 114 (1980) 105

4 J M Reın, W E van der Linden and $H$ Poppe, Anal Chim Acta, 126 (1981) 1

5 S D Kolev and E Pungor, Anal Chem, 60 (1988) 1700

6 S D Kolev and E Pungor, Anal Chım Acta, 208 (1988) 117,133

7 S D Kolev, K Tóth, E Lindner and E Pungor, Anal Chim Acta, 234 (1990) 49

8 R B Bird, W E Stewart and EN Lightfoot, Transport Phenomena, Wiley, New York, 1960

9 R A Greenkorn and D P Kessler, Transfer Operations, McGraw-Hıll, New York, 1972

10 M Masubuch, J Basic Eng, 82 (1960) 51

11 C J King, AIChE J , 10 (1964) 671

12 C J King, Ind Eng Chem, Fundam, 4 (1965) 125

13 J Szekely, Int J Heat Mass Transfer, 6 (1963) 833

14 R J Nunge, E W Porta and W N Gill, Chem Eng Symp Ser , 63 (1967) 80

15 S D Kolev and WE van der Linden, Anal Chım Acta, 247 (1991) 51

16 J M Kooljman, Chem Eng Scı, 28 (1973) 1149

17 V A Ditkın and P I Kuznetzov, Handbook on Operational Calculus, Gosudar Izd Techniko-Teoret Literat, Moscow, 1951 (in Russian)

18 J A Nelder and R Mead, Comput J , 7 (1964) 308

19 B Bernhardsson, E Martins and G Johansson, Anal Chım Acta, 167 (1985) 111

20 A P Hatton and A Quarmby, Int J Heat Mass Transfer, 5 (1962) 973

21 S D Kolev andd W E van der Linden, Anal Chim Acta, 257 (1992) 317

22 F B Hildebrand, Advanced Calculus for Applications, Prentice-Hall, Englewood Cliffs, NJ, 1963

23 G A Korn and T M Korn, Mathematical Handbook, McGraw-Hill, New York, 1968

24 S D Kolev and W E van der Linden, Num Heat Transfer, in press

25 S D Kolev and E Pungor, Anal Chım Acta, 194 (1987) 61 